\title{
ULTRASOUND IMAGING : SIGNAL ACQUISITION, NEW ADVANCED PROCESSING FOR BIOMEDICAL AND INDUSTRIAL APPLICATIONS
}

\author{
D. Kouamé, J.M. Grégoire, L. Pourcelot, J.M Girault, M. Lethiecq and F. Ossant
}

LUSSI, FRE 2448 CNRS University of Tours, France. E-mail: kouame@univ-tours.fr

\begin{abstract}
Use of ultrasound, namely in the biomedical diagnosis and industrial fields, pioneered in 1950s, is today particularly widespread. In the last decades, ultrasound imaging has benefited from advances in numerical technologies such as signal processing. On the other hand, the use of ultrasound imaging has increased the need for signal processing techniques. This paper presents a review and the up-to-date developments in ultrasound imaging techniques, including elementary principles, signal acquisition and processing, from one dimensional to multidimensional systems. This paper also deals with typical relevant applications.
\end{abstract}

\section{INTRODUCTION}

Due to its noninvasive and non ionizing nature and its flexibility, ultrasound (US) systems are a widely used modality for real time imaging. Though a high number of applications are met in the biomedical field, industry remains an area of important use. Ultrasound imaging is continuously growing in each of these fields. This is namely due to three reasons. The first one is linked to important advances in transducers (used for generation and detection of ultrasound) technology. The second is the improvement brought by advances in digital technologies, and namely advances in signal and image processing methods and technologies. The last one is the wide variety of applications in medical as well as in industrial areas. Concerning medical areas, for example, applications are as diverse as the different parts of the human body.

Utilization of Ultrasound imaging ranges from 1D to 4D applications. Obtaining high quality images for visualization or characterization purposes has been a big concern for long time. The first works were performed in 1D and concern the so-called A mode, that is the representation of ultrasound signal magnitude versus time. Alternatively, interrogating materials in one direction and displaying a single signal value over time as a scrolling display gives the so-called time motion (TM) mode. Generally speaking the 1D (US) signal processing is intended for quantitative extraction of a single parameter. This is typically the case for

Thanks to EC for funding. velocity estimation either through US Doppler spectrum estimation, US time of flight estimation or material or tissue characterization [1],[2]. Multi-dimensional signal or image, namely 2,3 or $4 \mathrm{D}$ processing, deals on one hand with improvement of image quality in terms of speckle processing, resolution, contrast enhancement and on the other hand with appropriate image visualization.

This paper provides an overview of the typical concepts of ultrasound imaging including up-to-date developments while describing some basic applications.

\section{ULTRASOUND SIGNAL ACQUISITION SYSTEMS}

All ultrasound systems consist of five parts as shown in Fig.1 : the first includes an acoustical part, the transducer, which performs both the generation and the detection of ultrasound. This transducer may consist of one element (which has to move) or of multiple elements for multidimensional signal acquisition. The typical working frequency depends on the materials investigated by ultrasound. These frequencies are basically less than $500 \mathrm{KHz}$ in air and range from $1 \mathrm{MHz}$ in some liquids or biological tissue up to 50 or $100 \mathrm{MHz}$ in some special applications. The second one is the electronic part which controls the US signal emission, beamforming, reception, and conditioning. The third and fourth parts are respectively US signal acquisition processing and display. And finally the fifth part is the material or tissue under consideration. The basic principle of US imaging consists in emitting US waves thanks to a transducer toward the material under consideration and receiving the reflected (echo)waves which result from the interaction between US waves and the material. The relevant signals are detected by the electronic part, conditioned, acquired, processed and displayed in an appropriate format. US image is in fact a collection of individual signals reflected from each interface inside the material. The exact processing depends on the US characteristics of the materials and the type of information required. However, the resulting signal or image quality that is its ability to restore information existing in the material, depends for a large part on the characteristics of the transducers such as central frequency and bandwidth. 


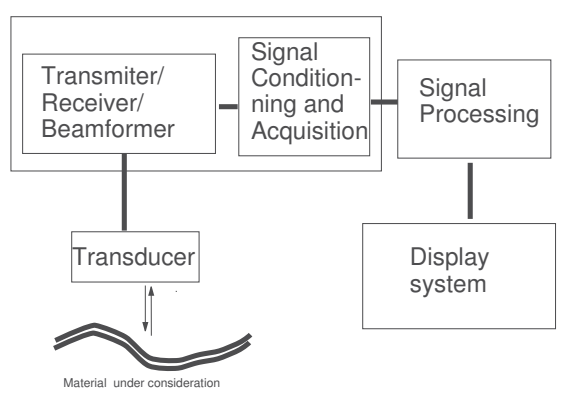

Fig. 1.

Ultrasound Image acquisition system

\section{ONE DIMENSIONAL SIGNAL PROCESSING : TIME, FREQUENCY AND VELOCITY ESTIMATION}

The primary aim of 1D US signal processing is to extract some parameters form the US signal for estimation or detection purpose. One important matter of concern is flow velocity estimation. Two kinds of techniques are used for this purpose.

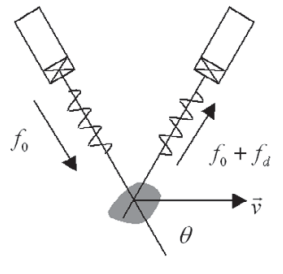

Doppler principle

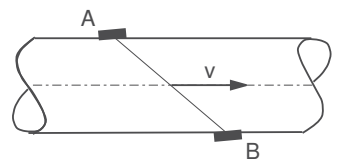

Time of flight principle
Fig. 2. Velocity estimation principles.

The first one is Doppler signal processing. Blood flow in vessels and pipe fluid flow in industrial applications can be accessed through analysis of the signal. Doppler signals are obtained by directing an ultrasound beam to a moving flow containing backscattering particles Fig.2. The wave reflected from the particle is slightly Doppler-shifted by the movement of the particles. After in-phase demodulation, the Doppler signal containing the Doppler frequency shift is used to extract flow velocity (which is proportional to Doppler frequency shift). Although the Doppler principle seems simple, in practice, the precise determination of the Doppler frequency is sometimes cumbersome because of physical phenomena that introduce inaccuracies in estimation of the velocity. Therefore, many trade-offs have been suggested. For example, in some real-time 2-D Doppler systems, the estimation of velocity is performed with only a few (8 or 16) samples to match the imaging frame rate. This has involved the development of fast, but rather inaccurate, algorithms. Other methods have been investigated when precise measurements of flow velocity are required,. One of the most used methods for this purpose is parametric (AR, ARMA,...) modelling. However, due to important limitations linked to model effective order change, alternative had to be proposed, [3].

The second one is the so-called time of flight technique. In this case the velocity estimation does not require the presence of particles in the flow. Transducers located on each side of the flow are used to acquire the signal from two areas (A and B for instance), Fig.2. The flow velocity is evaluated by estimating time of flight of the US in the fluid. Here, the velocity estimation is based either on narrow band or wide band correlation techniques,[4],[5].

These kinds of velocity estimations are used in industrial area namely for US flowmeters.

Other areas of interest of 1D US signal processing are related to media parameter estimation such that US attenuation or non-linear coefficient estimation through analysis of radio-frequency signals and their time frequency contents. Ultimately, rare event tracking also belongs to 1D US signal processing. This is the case for cerebro-vascular emboli detection. Emboli are foreign particles (fat, air,...) freely flowing with normal blood and that are able to obstruct blood flow in some regions. Their early detection is of great importance for diagnosis purpose. Here again, Doppler signal is used for that purpose and both time-frequency and knowledge-based techniques have been investigated and are still under development in many current works [6], [7].

\section{2D IMAGING}

US Image processing has been mainly centered on $2 \mathrm{D}$ image manipulation. Basically, 2D US imaging systems operate in Brightness-mode or B-mode Fig.3. A typical B-mode image is obtained from of a set individual radio-frequency (RF) signals by filtering, envelope detection and then logcompression,[8].

This kind of processing as well as the characteristics of the transducer and the materials may result in low resolution either axially (in the direction of the US beam) or laterally (in the perpendicular direction) through blurring effects contributing to a poor quality image. Improving 2D US image resolution is a major concern which has given rise to an important number of works. Many directions have been followed; the two main being novel transducer designs and signal processing improvement. Here, we deal with this last direction. If a part of works use B-mode images, a great number of works deal with RF signals since they contain the overall information available on the imaged material and 


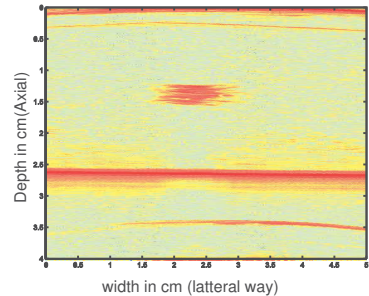

Original

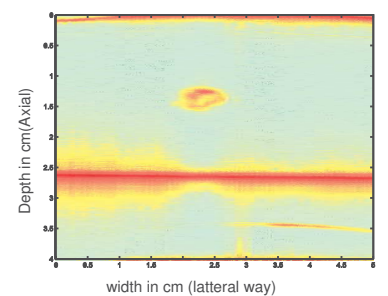

resolution improved using AR
Fig. 3. B-mode image of resolution improvement.

their improvement leads to improvement of B-mode images. The problem of 2D US image resolution improvement can be expressed in terms of convolution. The radio-frequency signal $y(n, m)$ can be expressed as :

$$
y(n, m)=h(n, m) * x(n, m)+\eta(n, m)
$$

where $n$ is the axial direction index and $m$ is the lateral one. $x(n, m)$ is the reflectivity function of an individual point of the imaged material, $h(n, m)$ is the system impulse response and $\eta(n, m)$ is an additive noise term. This convolution may be separated into two independent convolutions; one in the axial direction and another in the lateral direction.

Thus, the problem of US image resolution improvement and distorsion reduction comes to a problem of blind deconvolution in most works, [9],[10], the aim of which is to extract $x(n, m)$ from $y(n, m)$. Different types of approaches have been investigated. A number of studies were based on second or higher order statistics (HOS). It was shown, based on bispectrum estimation [11],[12], that (HOS) could be used to improve US image resolution under independent identically distributed assumption for $x$. This assumption does not hold in all practical situations. A better resolution was achieved using cepstrum parameter estimation and namely using generalized cepstrum,[13], [14] assuming separation between $x$ and $h$ in cepstral domain. This separation in turn does not always hold. Other investigations of deconvolution using both higher-order spectral methods and wavelet analysis [15], [16] were reported to significantly improve the quality of ultrasonic skin images. A quite interesting but simpler alternative to these methods, that we have introduced, is Autoregressive (AR) parameter modelling to improve the resolution. It consists in modelling the RF signal as the envelope on an AR process. This resulted in a substantial improvement of US image resolution.

Alternative 2D US imaging applications are linked to Doppler systems. Here, as opposed to section 3, movement detection may be considered in terms of color flow images. This color Doppler image, can be superimposed to a B-mode image in order to point out flow (of blood for instance) in the image structure. Classically, two main colors (blue and red) are used. Shadings of red are flows toward the transducer and and shadings of blue are flows away from the transducer; the Doppler frequency (or velocity) being evaluated by the brightness of the relevant color. Here, the contributions of signal processing techniques are those presented in section 3. However, the main techniques used for color flow mapping are based on either phase shift estimation or time shift estimation, see e.g. [17]. An alternative color flow imaging is the Power-mode Doppler(PMD). For this, instead of estimating the Doppler frequency and the relevant direction, the Doppler signal power is estimated. The main advantage of PMD is that it is easier to estimate and less dependent on angle than color Doppler. This technique may also be used for emboli detection [18]

An important emerging biomedical US imaging technique makes use of non-linear propagation. This has led to the so-called harmonic US imaging that produces images at harmonic frequency, for instance at $2 f_{0}$ if emitting US frequency is $f_{0}$. In some media, and namely in gas bubbles, the non linear behavior of US is particularly high. This has contributed to the development of so called Ultrasound contrast agents (USCA) and the relevant USCA imaging techniques, e.g [19],[20]. USCA are highly reflecting small gas bubbles in a liquid. Injected intravascularly, they strongly increase image contrast by increasing the intensity of signals reflected from blood-filled regions.

\section{3D AND 4D IMAGING}

Although 2D US imaging offers very acute understanding of the imaged media, its has some intrinsic limitations; the main of which being $2 \mathrm{D}$ viewing of $3 \mathrm{D}$ structures. This requires a mind reconstruction of actual 3D structures, which is in some situations difficult to achieve. A way to overcome this is to take multiple scans of some particular locations and to quantitatively analyze them afterwards. This is particularly time consuming. 3D and 4D may thus be used to overcome these limitations. 4D US imaging is real-time 3D US imaging in which time is taken as the fourth coordinate. The starting point of 3D/4D US imaging is, like in the $2 \mathrm{D}$ case, the acquisition system. The basic principle consists in the acquisition of a volume data set. Most of current methods consist in acquiring a series of $2 \mathrm{D}$ images in a volume of interest while moving the transducer(which is of course a specific transducer) using a motor. This implies additional techniques for processing a slice within the volume and taking into account the motion when imaged objects move. Many relevant imaging techniques have been developed recently. Due to pages limitation, the readers are referred e.g. to [21],[22], [23]. One important use of these techniques is currently the field of "telemedicine" which associates the previous techniques embedded in lightweight devices and data transmission in order to perform diagnosis 
or monitoring at distance.

\section{CONCLUSION}

This paper has reviewed the main ultrasound imaging systems and techniques from 1D signals to 4D images. The part played by signal and image processing has become larger and larger, and current trends in new applications, both in industrial and biomedical fields, are a sign that this part will keep on growing. Finally, several practical developments have been presented and also discussed during the oral presentation in this special session.

\section{REFERENCES}

[1] S. Satomura, "Ultrasonics doppler method for the inspection of cardiac function," J. Acoust. Soc. Am., vol. 29, pp. 1180-1185, 1957.

[2] P.N.T. Wells, Physical principle of Ultrasonics Dignosis, London Academic Press, 1969.

[3] D. Kouamé, J.M. Girault, and F. Patat, "High resolution processing techniques for ultrasound doppler velocimetry in presence of colored noise part 1 : Nonstationary methods," IEEE Trans. on Ultr. Fer. Freq. Control, vol. 50, pp. 257-266, 2003.

[4] Lora C. Weiss, "Time varying system characterization for wideband input signal," Signal processing, vol. 55, pp. 295-304, 1996.

[5] K. C. Ho and Y. T. Luo, "Optimum discrete scaling and its application to delay and doppler estimation," IEEE Trans. Sig. Proc, vol. 46, pp. 904-916, 1995.

[6] A.R. Naylor L. Fan, D.H. Evans, "Automated embolus identification using a rule-based expert system," $U l$ trasound Med. Biol, vol. 27, pp. 1065-1077, 2001.

[7] D. Kouamé, M. Biard, J-M. Girault, A. Bleuzen, F. Tranquart, and F. Patat, "Statistical and neurofuzzy approaches for emboli detection," in Proceedings of 12th European Signal Processing Conference EUSIPCO, 2004.

[8] M. Fatemi and A.C. Kak, "Ultrasonic b-scan imaging: Theory of image formation and a technique for restoration," Ultras. Imaging, vol. 2, pp. 1-47, 1980.

[9] E.E. Hundt and E.A. Trautenberg, "Digital processing of ultrasound data by deconvolution," IEEE Trans. Son. Ultras., vol. 27, pp. 249-252, 1980.

[10] J.A. Jensen, "Deconvolution of ultrasound images," Ultras. Imaging, vol. 14, pp. 1-15, 1992.
[11] U. R. Abeyratne, A.P. Petropulu, and J.M. Reid, "High order spectra based deconvolution of ultrasound images," IEEE Trans. Ultr. Ferr. Freq. Cont., vol. 42, pp. 1064-1075, 1995.

[12] U. R. Abeyratne, A.P. Petropulu, J.M. Reid, T. Golas, E. Conant, and F. Forsberg, "High order vesus second order statisticsin ultrasound images deconvolution," IEEE Trans. Ultr. Ferr. Freq. Cont., vol. 44, pp. 1409-1416, 1995.

[13] T. Taxt, "Comparison of cepstrum-based methods for radial blind deconvolution of ltrasound images," IEEE Trans. Ultr. Ferr. Freq. Cont., vol. 44, pp. 1409-1416, 1995.

[14] T. Kobayashi and S. Imai, "Spectral analysis using generalized cepstrum," IEEE Trans. Acoust. Speec. Sig. Proces., vol. 32, pp. 1087-1089, 1984.

[15] R. Neelamani, H. Choi, and R. Baraniuk, "Wavelet deconvolution for ill conditionned systems," in IEEE Int. Conf. Acoust. Speec. Sig. Proces(ICASSP), 1999.

[16] S. Wan, B. I. Raju, and M. A. Srinivasan, "Robust deconvolution of high frequency ultrasound images using higher ordre spectral analysis and wavelets," IEEE Trans. Ultr. Ferr. Freq. Cont, vol. 50, pp. 1286-1295, 1984.

[17] J.A. Jensen, Estimation of Blood Velocities using Ultrasound: A signal processing approach, Cambridge University Press, 1996.

[18] M.A. Moehring and M.P. Spencer, "Power m-mode doppler for observing cerebral blood flow and tracking emboli," Ultras. in Med.\& Biol., vol. 28, pp. 49-57, 2002.

[19] Barry B. Goldberg, Ji-Bin Liu, and Flemming Forsberg, "Ultrasound contrast agents: A review," Ultras. in Med.\& Biol., vol. 20, pp. 319-402, 1994.

[20] M. Postema, A. Van Wamel, C.T. Lancee, and N. De Jong, "Ultrasound-induced encapsulated microbubble phenomena," Ultras. in Med.\& Biol., vol. 30, pp. $827-$ 840, 2004.

[21] W. Lees, "Ultrasound imaging in three or four dimension," Semin. Ultrasound, CT and MRI, vol. 22, pp. 85-105, 2001.

[22] T. R. Nelson and D.H. Pretorius, "Three dimension ultrasound imaging," Ultrasound in Med. \& Biol., vol. 24, pp. 1243-1270, 1998.

[23] A. Fenster and D.B. Downey, "3-d ultrasound imaging : a review," IEEE Trans. Biom Engin., vol. 15, pp. 41$51,1996$. 